\title{
Voyages, rencontres, échanges au XVII siècle. Marseille carrefour, édité par S. REQUEMORA-GROS
}

\section{Monica Pavesio}

\section{CpenEdition \\ Journals}

\author{
Edizione digitale \\ DOI: $10.4000 /$ studifrancesi. 16345 \\ ISSN: 2427-5856 \\ Editore \\ Rosenberg \& Sellier

\section{Edizione cartacea} \\ Data di pubblicazione: 1 juillet 2019 \\ Paginazione: 157-158 \\ ISSN: 0039-2944
}

URL: http://journals.openedition.org/studifrancesi/16345

\section{Notizia bibliografica digitale}

Monica Pavesio, "Voyages, rencontres, échanges au XvI e siècle. Marseille carrefour, édité par S. REQUEmoRAGRos», Studi Francesi [Online], 187 (LXIII | I) | 2019, online dal 01 juin 2019, consultato il 25 janvier 2021. URL: http://journals.openedition.org/studifrancesi/16345; DOI: https://doi.org/10.4000/studifrancesi. 16345

Questo documento è stato generato automaticamente il 25 janvier 2021.

\section{(c) (i) (9)}

Studi Francesi è distribuita con Licenza Creative Commons Attribuzione - Non commerciale - Non opere derivate 4.0 Internazionale. 


\title{
Voyages, rencontres, échanges au XVII siècle. Marseille carrefour, édité par S. REQUEMORA-GROS
}

\author{
Monica Pavesio
}

\section{NOTIZIA}

Voyages, rencontres, échanges au XVII siècle. Marseille carrefour, édité par S. REQUEMORA-GROS, Tübingen, Gunter Narr, 2017, «Biblio 17» 211, 575 pp.

1 Il volume raccoglie una selezione di contributi presentati al $43^{\circ}$ convegno della North American Society for Seventeenth-Century French Literature, organizzato in onore del Professor Pierre Ronzeaud, presso l'Università di Aix-Marseille nel 2013.

2 La scelta di celebrare Marsiglia e la Provenza nel convegno annuale della società di secentisti americani nasce dalla volontà di analizzare, da un punto di vista storico, letterario, politico, scientifico, religioso, musicale e iconografico, gli scambi, i passaggi, i viaggi che, nel xvII secolo, hanno reso la città e la regione un carrefour, una luogo di circolazione di uomini, una terra di incontri, di partenze e di arrivi.

3 I quarantuno contributi riuniti nel volume sono suddivisi in dieci sezioni che presentano Marsiglia sotto differenti punti di vista e categorie.

4 La prima sezione, intitolata «Voyages», raccoglie nove comunicazioni incentrate sulle relazioni di viaggio, reali o immaginarie, burlesche o galanti, che illustrano e approfondiscono gli incontri commerciali e culturali tra Oriente, Europa e Occidente. Dopo la prima comunicazione di P. RONZEAUD dedicata alle figure dei viaggiatori / narratori francesi delle utopie letterarie del XviI secolo (Voyager dans les récits de voyages imaginaires $d u$ xvIIe 
siècle: pourquoi pas?M.CH. PIOFFETLe théâtre du Nouveau Monde: dialogues franco-amérindiens dans les écrits viatiques de la Nouvelle-France,S. A. MELZERComment écrire l'histoire de la rencontre franco-amérindienne?

I saggi successivi di K. DESPLANQUE (Un miroir sur le regard miroité: "Le Voyage d'Encausse" et le récit de voyage humoristique, pp. 73-81), di J. LECLERC (Ulysse en Provence: voyages, temporalité et intertextualité burlesques, pp. 83-92), di C. NÉDELEC (Voyager en galant homme, pp. 93-102), di м. BEDEL (Marseille: porte de l'Inde. Récit du médecin curieux devenu l'explorateur privilégié d'un ailleurs fabuleux, pp. 103-115), di v. Joucla (Dissuader le lecteur de voyager: le curieux projet des "Mémoires des voyages du sieur André Demarez d'Avignon", pp. 117-126) e di I. APоSTOLou (Le voyage du comte de Marcheville de Marseille à Constantinople: tradition et nouveauté, pp. 127138) analizzano alcuni récit de voyage secenteschi curiosi e poco conosciuti.

seconda sezione, "Échanges et mobilité en Méditerranée», meno corposa della precedente, riunisce tre comunicazioni incentrate sugli scambi commerciali nel Mar Mediterraneo. A. BACCAR BOURNAZ, (Acteurs et enjeux au large des côtes provençales d'après «La Gazette de France» (1640-1670), pp. 141-150) incentra la sua analisi sugli scambi nella parte occidentale del Mediterraneo, mentre i viaggi e i conflitti nella parte orientale vengono descritti nei due saggi di J.P. FARGANEL, (Consuls, marchands et voyageurs français dans le Levant aux XVIIe

et XVIII siècles, une vision de soi et de l'altérité: d'un stéréotype l'autre,O. SENNOUNE,Les Marseillais dans l'Echelle d'Alexandrie au XVII siècle. Entre un cadre de vie exotique et un nouveau modèle

Quattro saggi compongono la terza sezione, denominata "Correspondances», incentrata sugli scambi epistolari. Il primo di D. REGUIG è dedicato alle lettere di Boileau ( $\mathrm{La}$ correspondance de Boileau; un art de la distance, pp. 177-188), mentre gli altri tre sono incentrati sulla corrispondenza di Mme de Sévigné con la figlia, trasferitasi in Provenza (B. LANDRY, Madame de Sévigné et le 'Fourbin' de Marseille, pp. 189-200), sui suoi viaggi a Marsiglia (N. FREIDEL, Mme de Sévigné à Marseille: promenade romanesque ou mission politique?, pp. 201-210), e sulla descrizione, o piuttosto, sulla non descrizione della Provenza nelle lettere della grande romanziera e di Voiture (s. RoLLin, La Provence et la province dans les lettres de Mme de Sévigné et de Voiture: les enjeux d'une écriture oblique, pp. 211-222).

8 La città di Marsiglia, come punto d'incontro e di scambi, è la protagonista del quarto gruppo di comunicazioni, riunite sotto il titolo di «Marseille, carrefour des savoirs et des échanges de savoir». Il primo articolo di J. R. ARMOGATHE (De Phocée à Marseille: les antiquités marseillaises dans l'historiographie des XVIIe et XVIII siècleE, BURYLa Provence, carrefour de la République des sciences à l'âge de la révolution scientifique: Peiresc et GassendiM. MARRACHE-GOURAUDMerveilles à l'encan, séductions d'une ville: Marseille dans la culture européenne de la curiositéxVIIITREVISANI-MOREAUDe la flore locale au carrefour des savoirs: "L'Histoire des plantes qui naissent aux environs d'Aix et dans plusieurs autres endroits de la Provence" par Pierre-Joseph Garidels. HOUDARDLes écritures du diable: impressions et contagions de la possession d'Aix-en-Provence dans le premier XVII siècleV. JULLIENGassendi à Marseille, qu'allait-il faire dans cette galère?

9 Il quinto gruppo di studi, riuniti con il titolo di «Espaces religieux», è collegato agli scambi religiosi in Provenza. A. RÉGENT-SUSINI analizza gli scritti del Père Coton, 
missionario nel sud della Francia, (De l'oral à l'écrit: les "Sermons sur les principales et difficiles matières de la foi" du Père Coton 'réduits par l'auteur en forme de Méditations', ou l'usage du paragraphe dans le livre de dévotion, pp. 321-336); C. VENNER si occupa delle poesie cristiane di A. Godeau, vescovo di Grasse (Les îles de Lérins dans les "Poësies chrestiennes" d'Antoine Godeau, pp. 337-348); sono dedicati a Maria Maddalena che, secondo la tradizione provenzale, si fermò a Marsiglia, l'articolo di C. MCCALL PROBES sugli scritti dei poeti del cenacolo d'Henri d'Angoulême (La Sainte-Baume et la Madeleine chez les poètes du cénacle aixois d'Henri d'Angoulême: vers une rhétorique du paysage et de l'heureuse pécheresse, pp. 349-360) e il successivo di Р. сномÉTY sull'opera di Pierre de Saint-Louis (L'Eliade, ou l'odyssée du savoir: science et poésie chez Pierre de Saint-Louis, pp. 361-372).

Dagli scambi religiosi si passa a quelli politici nella sesta sezione, intitolata «Espaces de pouvoirs», che analizza l'opposizione tra potere centrale e province. T. PARKER nel suo saggio (Bouillabaisse et Cassoulet: aux origines d'une question esthétique, politique et identitaire, pp. 375-385) riflette sui legami tra le cucine regionali e l'identità nazionale francese; M. ROSSELLINI si sofferma sull'opposizione tra cattolicesimo e protestantesimo nei Mémoires di un prigioniero ugonotto (Marseille et la Provence dans les "Mémoires" du galérien Jean Marteilhe, pp. 387-398); M. CL. CANOVA-GREEN rivolge il suo interesse alle entrate solenni di Luigi XIII nelle città provenzali appena conquistate ( $L a$ ville, la province et le roi. Les entrées provençales de Louis XIII à l'automne 1622, pp. 399-409).

11 Il settimo gruppo di comunicazioni («Rencontres dans les arts du spectacle») è dedicato al teatro, e più in particolare, alla rappresentazione sulle scene francesi di Marsiglia $\mathrm{e}$ della Provincia come luoghi esotici. I primi due contributi di S.H. FLECK (La comédie-ballet et les mondes fictionnels, pp. 413-421) е di В. РОRОт (Étrangers et voyageurs dans les ballets et les comédies-ballets de Lully (1655-1670), pp. 423-442) analizzano l'esotismo nelle comédiesballets. Il terzo di M. DESPREZ (Du texte de "conjointure» dans la constitution du comédien professionnel: le cas du "Prologue de La Porte, comédien à Bourges, contre les Jésuites", pp. 443-452) si occupa del genere del prologo drammatico; il quarto di M. GROss (Le Spectateur en vue: les voyages européens de Joseph Furttenbach, Andrea Gryphius et Gottfried Wilhelm Leibniz, pp. 453-468) dei viaggi in Europa di eruditi tedeschi.

Dal teatro si passa alla musica nell'ottava sezione intitolata «Rencontres musicales» con i tre articoli di M. FolLIARD sui balletti creati da Théophile de Viau per Luigi XIII (Le lieu de l'autre. Les vers pour ballet de Théophile (1617-1626), pp. 469-485); di T. VERNET sul soggiorno pedagogico a Marsiglia di due giovani principi reali ( $(V o u d r i a n$ n'ooublidar ren per tachar de vous plaïré», le séjour marseillais des ducs de Berry et de Bourgogne en mars 1701, pp. 487-496); di C. CESSAC sulle opere del musicista provenzale Mouret ("Les Festes de Thalie" de Jean-Joseph Mouret ou comment un musicien provençal fait basculer l'opéra-ballet dans la comédie lyrique, pp. 497-506).

La nona sezione è riservata alle «Rencontres littéraires» con due studi dedicati a due romanziere secentesche e alle descrizioni, nelle loro opere, di viaggi e paesaggi nel sud della Francia: il primo di P. GETHNER è incentrato sulla contessa di Murat (Voyages réels et irréels dans "Voyage de campagne", pp. 509-517); il secondo di J. vos-CAMY ad Antoinette de Salvan de Saliès (Antoinette de Salvan de Saliès, une muse albigeoise, pp. 519-529). L'articolo successivo riguarda, invece, i viaggi e i viaggiatori nelle riscritture del mito di Fedra (м. BRUNEL, De l'intrigue romanesque à la tragédie de l'errance: le voyage et les voyageurs dans "Phèdre" et ses réécritures (Euripide, Sénèque, Garnier, Gilbert, Pradon, Racine), pp. 531-542). 
14 L'ultimo gruppo di studi si occupa di Rencontres iconographiques, con due saggi sugli scambi artistici tra Genova e Marsiglia (s. CHABRE, Les échanges artistiques entre Gênes et Marseille: la commande de sculptures génoises en Provence (1620-1730), pp. 545-558) e tra Parigi e Marsiglia (M. THÉRON, De Paris à Marseille, les projets de décor de la première Réale de Louis XIV: Le Brun \& Tuby versus Girardon, pp. 559-575). 